\title{
Status of the FP420 Project at the LHC
}

\author{
Andrew D Pilkington * \\ Department of Physics and Astronomy, The University of Manchester, \\ Manchester M13 9PL, UK.
}

\begin{abstract}
A brief motivation for the installation of forward detectors in the $420 \mathrm{~m}$ region from the ATLAS/CMS detectors is presented and the status of the FP420 R\&D project is reviewed.
\end{abstract}

\section{Introduction}

FP420 is an R\&D collaboration that proposes to install forward detectors $420 \mathrm{~m}$ either side of the interaction point at ATLAS and/or CMS [2]. The purpose of the detectors is to tag the protons from the central exclusive process $p p \rightarrow p+X+p[3]$, where $X$ is a centrally produced system separated by a large rapidity gap from the outgoing protons. For central masses of approximately $120 \mathrm{GeV}$, the protons typically emerge from the beam in the large dispersion region at $420 \mathrm{~m}$ from the interaction point. This makes FP420 a desirable upgrade for light Higgs boson searches at the LHC.

The central exclusive process is attractive for two reasons. Firstly, the protons are typically scattered through small angles and, to a good approximation, the central system is produced in a $0^{++}$state. This allows the determination of the quantum numbers of any observed resonance. Secondly, because of the exclusive nature of the event, the mass of the central system can be reconstructed from just the outgoing proton momenta using the so-called missing mass method [4]. This allows the mass of any resonance to be determined to an accuracy of approximately $2 \mathrm{GeV}$, regardless of the decay mode of the particle.

The focus of attention in this area has been on the discovery of a Higgs boson at the LHC. The CEP Standard Model Higgs boson is predicted to be observed with $30 \mathrm{fb}^{-1}$ of delivered luminosity in the $\mathrm{WW}^{*}$ channel if the Higgs boson has a mass of $140<M_{h}<200 \mathrm{GeV}$ [5]. The $b \bar{b}$ decay channel of a Higgs boson has also attracted a great deal of interest because the QCD $b \bar{b}$ background is strongly suppressed by the spin-selection rules. Recently, high $\tan \beta$ MSSM scenarios have been investigated with the conclusion that the $b \bar{b}$ channel will be observable up to $m_{h} \sim 160 \mathrm{GeV}$. For such scenarios, the Higgs boson will be observed at the LHC (and possibly the Tevatron) in the $\tau \tau$ decay channel. However, the excellent mass resolution of FP420 would provide additional insight into the observations. Finally, the possibility of investigating the CP structure of the Higgs sector is possible at the LHC by measuring the azimuthal asymmetries of the outgoing protons [6]. Such a measurement was previously thought to possible only at a linear collider.

The proposal of FP420 has been boosted by the indication that the exclusive process is currently being observed in the CDF data. Using the Khoze, Martin and Ryskin (KMR) calculation for central exclusive production on which the majority of LHC predictions are based [7], it was predicted in [8] that an excess of events would be obervable in the DPE di-jet data at CDF. Preliminary results imply that this is in fact the case [9]. Furthermore, and significantly, CDF observe a reduction in the fraction of $c$ and $b$-quark jets in the region where the excess exists. This is a direct prediction of the KMR model. CDF have also

*On behalf of FP420. 
performed a search for central exclusive $\gamma \gamma$ production and observe 2 candidate events [10], which is also consistent with the KMR predictions.

\section{$2 \quad$ FP420}

FP420 is a magnetic spectrometer. The protons that emerge from the beam in the $420 \mathrm{~m}$ region are tagged and the position of the proton relative to the beam depends primarily on the fractional momentum loss of the proton during the interaction. Detailed investigation has been carried out using chromaticity grids to relate the position and angle of the proton measured by FP420 to the energy and angle of the proton at the interaction point.

The first task of FP420 was to design a new $15 \mathrm{~m}$ section to replace the interconnection cryostat in the $420 \mathrm{~m}$ region. This interconnection cryostat, which will be present at the start-up of the LHC, is responsible for providing the continuity of the $2 \mathrm{~K}$ beam pipes, the insulation vacuum, the electrical power, the cryogenic circuits and the thermal and radiation shielding. This continuity must be retained, whilst providing access to warm beam pipes. The re-design has been achieved mainly by using existing LHC components.

The proton position measurements will be made by two 3D silicon detector stations placed at each end of the FP420 region. 3D silicon is radiation hard and capable of withstanding the large particle fluxes that will be experienced by FP420. Furthermore, with the $3 \mathrm{D}$ silicon design, there is only a $5 \mu \mathrm{m}$ dead region allowing proton measurements with an almost edgeless detector. The position measurement within the silicon can be made to $10 \mu \mathrm{m}$. The readout of the $3 \mathrm{D}$ silicon readout is achieved by using the standard ATLAS pixel readout chips.

The detectors will be moved closer to the beam once the beam has stabilised. The chosen movement mechanism is the Hamburg pipe, with the detectors rigidly fixed to the side of the beampipe and the pipe itself moved to place the detectors near to the beam. The standard beam pipe is replaced by a beam pipe with a pocket, to which the detector stations are attached. This allows the protons to pass through a small window of approximately $300 \mu \mathrm{m}$, which reduces the probability of particle showering. The measurement of the detector station position relative to the beam will be made with beam positioning monitors (BPMs). It is expected that this measurement will be accurate to $50 \mu \mathrm{m}$ and benchtests are currently being performed. Investigations into the electromagnetic interaction between the beam and the re-shaped beam pipe have been completed [11]. A series of benchtests and simulations were performed, with the conclusion that the installation of FP420 will have a negligible contribution to the total impedance budget of the LHC.

The detector stations will be instrumented with quartz and gas Cherenkov timing detectors, which are capable of measuring the proton time-of-flight to an accuracy of 10ps. This enables an event vertex to be reconstructed from the difference in proton time of flight. The vertex reconstruction is necessary to remove backgrounds from events constructed from more than one proton-proton interaction. The vertex of the hard scatter can be matched to the vertex reconstructed from the proton TOF and it is expected that $95 \%$ of backgrounds from the pile-up of proton-proton interactions can be rejected by this technique.

Finally, studies into machine induced backgrounds from beam-halo, beam-gas and secondary showering have been performed. Beam-halo backgrounds from both betatron and momentum collimation have been investigated, with the conclusion that the proton rate is negligible if the detectors are approximately $5 \mathrm{~mm}$ from the beam. The background from proton hits in FP420 from beam-gas inelastic scattering has also been found to be negligi- 
ble. Secondary showering occurs due to proton transport from the interaction point. The protons interact with machine elements upstream of the detectors and produce a shower of particles (mainly neutrons and photons) in the FP420 detectors. The background rate from secondary showers is currently under investigation.

\section{Summary}

Instrumenting the LHC with forward detectors capable of measuring proton from central exclusive production can extend the physics potential of the ATLAS and CMS detectors. The FP420 R\&D project to install such detectors is progressing well with many technical issues overcome. Studies are ongoing and those completed indicate that the search for central exclusive production is achievable at the LHC.

\section{References}

[1] Slides: http://indico.cern. ch/contributionDisplay.py? contribId=99\&sessionId=7\&conf Id=9499

[2] B. E. Cox, arXiv:hep-ph/0609209.

[3] A. De Roeck, V. A. Khoze, A. D. Martin, R. Orava and M. G. Ryskin, Eur. Phys. J. C 25 (2002) 391 [arXiv:hep-ph/0207042].

[4] M. G. Albrow and A. Rostovtsev, arXiv:hep-ph/0009336.

[5] B. E. Cox et al., Eur. Phys. J. C 45, 401 (2006) [arXiv:hep-ph/0505240].

[6] J. R. Ellis, J. S. Lee and A. Pilaftsis, Phys. Rev. D 71, 075007 (2005) [arXiv:hep-ph/0502251].

[7] J. R. Forshaw, arXiv:hep-ph/0508274.

[8] B. E. Cox and A. Pilkington, Phys. Rev. D 72, 094024 (2005) [arXiv:hep-ph/0508249].

[9] C. Mesropian [CDF Collaboration] DIS2007, these proceedings.

[10] T. Aaltonen et al. [CDF Collaboration], arXiv:0707.2374 [hep-ex].

[11] Appleby, Jones and Roncarolo, Beam coupling impedance simulations and measurements for the LHC FP420 detector, PAC07 Conf. Proc. Albequeque, US, June 25-29 2007. 